\title{
Correction to: Long QT Syndrome
}

\author{
Andrew Krahn, Wael Alqarawi, and Peter J. Schwartz
}

\section{Correction to:}

M. Green et al. (eds.), Electrocardiography of Inherited

Arrhythmias and Cardiomyopathies, https://doi.org/10.1007/978-3-030-52173-8_1

The book was inadvertently published with an incorrect spelling of Chapter 1 author name.

The author name has now been corrected to "Peter J. Schwartz".

The updated version of this book can be found at https://doi.org/10.1007/978-3-030-52173-8_1 